\title{
DEFINIÇÃO DO CONTEÚDO DA MNEMÓNICA POSICIONAR
}

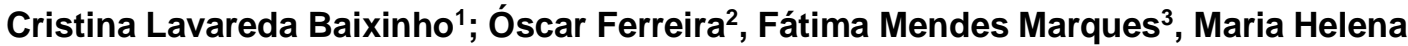 \\ Presado $^{4}$, Mário Cardoso ${ }^{4}$ e Helga Rafael ${ }^{6}$
}

\author{
${ }^{1}$ Escola Superior de Enfermagem de Lisboa, ciTechCare Portugal. crbaixinho@esel.pt \\ ${ }^{2,3,4}$ Escola Superior de Enfermagem de Lisboa, Portugal. ${ }^{2}$ oferreira@esel.pt; ${ }^{4}$ fmarques@esel.pt, ${ }^{4} \mathrm{mhpresado@esel.pt}$ \\ ${ }^{5} \mathrm{mmcardoso@esel.pt,}{ }^{6} \mathrm{hrafael} @ e s e l . p t$
}

\begin{abstract}
Resumo. Introdução: A Terapêutica de Posição é uma terapêutica essencial na prestação de cuidados de saúde, coadjuvante no tratamento de várias morbilidades, importante para a promoção do conforto e bem-estar em pessoas de maior fragilidade, auxiliando na prevenção de várias complicações decorrentes da doença e/ou tratamento. Objetivo: Definir os indicadores da Mnemónica - POSICIONAR, para futura validação por um painel de peritos. Método: Estudo de abordagem mista, desenvolvido em 3 fases. Apenas nos centramos na abordagem qualitativa do segundo estudo. Participaram 9 enfermeiros peritos que responderam, em duas rondas, a dois formulários, que foram sujeitos a análise temática de conteúdo segundo Bardin. Resultados: A análise de conteúdo permitiu a organização de um conjunto de intervenções para as 10 categorias definidas a priori e que são representativas da terapêutica de posição. Conclusões: O instrumento proposto, possibilita sistematizar um conjunto de critérios a utilizar na avaliação e promoção da segurança da pessoa a posicionar, bem como, facilitar a comunicação entre os profissionais de saúde e a continuidade de cuidados.
\end{abstract}

Palavras-chave: Posicionamento; Segurança do Doente; Cuidados de Enfermagem; Investigação Qualitativa.

\section{CONTENT DEFINITION OF THE MNEMONIC POSITION}

Abstract. Introduction: Position Therapy is an essential therapy to provide health care and to support the treatment of various morbidities, it's also important for the promotion of comfort and well-being in people of greater fragility, helping to prevent various complications resulting from the disease and / or treatment. Goal: To define the indicators for the Mnemonic - POSITION, for future validation by a panel of experts. Method: Study with a mixed approach, developed in 3 phases. We only focus on the qualitative approach of the second study. Participated 9 expert nurses who responded, in two rounds, to two forms, which were thematic content analysis according to Bardin. Results: Content analysis allowed the organization of a set of interventions for the 10 categories defined a priori and which are representative of the position therapy. Conclusions: The proposed instrument makes it possible to systematize a set of criteria to be used in assessing and promoting the safety of the person to be positioned, as well as facilitating communication between health professionals and continuity of care.

Keywords: Positioning of the Patient; Patient Safety; Nursing Care; Qualitative Research.

\section{INTRODUÇÃO}

Quando a pessoa não se consegue mover e posicionar na cama, é necessário garantir um posicionamento correto e para tal, usar técnicas de mobilização e de posicionamento específicos (Källman et al., 2013; Santos, Ferreira, \& Baixinho, 2017). Posicionar é uma intervenção que implica colocar o corpo numa posição diferente daquela em que se encontrava, de forma a redistribuir a pressão exercida sobre uma determinada parte do 
corpo (Moore, Cowman \& Conroy, 2011; Pickenbrock, Ludwig, \& Zapf, 2017), respeitando os princípios da biomecânica, que incluem o alinhamento corporal, o equilíbrio, o movimento, a fricção/atrito e a força mecânica (OE, 2013; Prezado et al., 2015).

O posicionamento terapêutico é uma intervenção muito utilizada nos cuidados de saúde, que implica a apreciação complexa da situação clínica e da pessoa, e uma decisão direcionada para a(s) finalidade(s) terapêutica(s) (Pickenbrock, Ludwig, \& Zapf, 2017). Apesar de ser frequente na clínica e dos seus efeitos serem complexos e importantes para a prestação de cuidados de saúde, partilhamos a opinião de que esta intervenção tem sido pouco valorizada na prática clínica, na formação e na investigação (Silva, \& Nascimento, 2012; Baixinho \& Ferreira, 2016; Schutt, Tarver, \& Pezzani, 2018; Santos, Ferreira, \& Baixinho, 2019).

Silva e Nascimento (2012) alegam que as mudanças de decúbito, realizadas pela equipa de enfermagem sob prescrição ou indicação do enfermeiro, envolve mais fatores do que simplesmente mudar a posição da pessoa na cama, já que a sua influência na mecânica pulmonar tem um efeito terapêutico importante.

A necessidade de avaliar uma multiplicidade de fatores e de antever as possíveis complicações decorrentes da não aplicação ou utilização incorreta desta terapia alerta para a necessidade de o plano terapêutico (programa de terapia de posição) ser individualizado, não apenas face ao desenvolvimento, ao grau de atividade e mobilidade da pessoa, mas também, face à forma como se posiciona ou é posicionada pelos cuidadores informais de modo a evitar consequências decorrentes de um posicionamento incorreto (Baixinho, Ferreira, Rafael \& Ferraz, 2016).

A apreciação da pessoa para a implementação desta terapêutica é um processo rigoroso que implica avaliar o estado cognitivo, os sinais vitais, a motricidade, a amplitude articular, a postura e alinhamento, os antecedentes pessoais de saúde, a situação clinica atual, o nível de independência, o nível de conforto, o peso, a altura e a determinação do risco de desenvolver lesões por pressão (Spruce, \& Van Wicklin, 2014; Pickenbrock, Zapf, \& Dressler, 2015; Baixinho, Ferreira, Rafael \& Ferraz, 2016). Todavia a avaliação da pessoa não esgota estes itens. Nas situações de saúde mais complexas pode haver necessidade de apreciar outros aspetos como a presença de feridas, drenos, dispositivos médicos, entre outros, que condicionem o posicionamento. 
A análise da situação deve ainda prever o contexto e a disponibilidade de recursos materiais como superfícies de apoio, camas articulares, almofadas e outros dispositivos de posicionamento e de ajuda ao mesmo, bem como económicos e consequentemente a possibilidade e o interesse da pessoa e ou família em os adquirir (Pickenbrock, Ludwig, \& Zapf, 2017; Schutt, Tarver, \& Pezzani, 2018).

Sendo uma etapa complexa, que exige "gastos" em tempo para ser efetuada com uma determinada periodicidade - mudanças de decúbito de 2 em 2 horas (Schutt, Tarver, \& Pezzani, 2018), não pode ser ultrapassada pelos riscos associados a uma má decisão na utilização desta terapêutica e aos consequentes custos que poderá acarretar para a pessoa cuidada, sua família e sociedade, nomeadamente pelo aparecimento de lesões por pressão (Källman et al., 2013; Santos, Ferreira, \& Baixinho, 2017).

Por outro lado, a decisão sobre esta terapêutica deve prever a antecipação de um conjunto de complicações que podem surgir durante a sua aplicação. A mobilidade funcional pode estar alterada em consequência de determinadas patologias, lesões ou cirurgias e revestese de um caráter negativo com repercussões reconhecidas (OE, 2013).

Por isso, os compromissos funcionais presentes em muitas pessoas devem ser considerados na tomada de decisão sobre o decúbito a selecionar e a sua finalidade, não devendo agravar as complicações decorrentes do processo de imobilidade tais como, entre outras, o encurtamento muscular e a rigidez articular (Källman et al., 2013; Santos, Ferreira, \& Baixinho, 2019)

Enquanto intervenção, a terapêutica de posição deve responder às necessidades da pessoa a que se destina, devendo prever a heterogeneidade das características dos clientes dos cuidados de saúde, a etapa vital em que se encontram e a diversidade de contextos onde são prestados os cuidados (Baixinho, Ferreira, Rafael \& Ferraz, 2016).

Pela importância de que se reveste e por ser uma área com pouca investigação, foi proposta a Mnemónica - POSICIONAR, para sistematizar um conjunto de critérios a utilizar na avaliação e promoção da segurança da pessoa e de forma a facilitar a comunicação entre os profissionais de saúde concretamente: a apreciação da individualidade da Pessoa a posicionar; a necessidade de Observação da pele de forma sistemática diariamente; a importância de Selecionar superfícies de apoio adequadas à pessoa a posicionar; de Identificar objetivos da terapêutica de posição adequando-os às necessidades do 
cliente; a necessidade de Capacitar para o posicionamento não só a pessoa que necessita de TP, como também o familiar cuidador; o Implementar medidas de conforto com vista a manter e ou aumentar o bem-estar da pessoa a posicionar; Obedecer aos princípios de biomecânica no que respeita ao alinhamento corporal, à postura, à força e à fricção; Negociar posições e decúbitos com a pessoa a posicionar de acordo com as suas preferências e com o familiar cuidador conforme as suas capacidades; Adequar as almofadas e os dispositivos de posicionamento para garantir uma TP em segurança e com conforto e Reavaliar a eficácia do posicionamento de forma a adequar o programa de TP à pessoa que dele necessita (Baixinho, Ferreira, Rafael \& Ferraz, 2016).

É objetivo deste estudo definir os indicadores da Mnemónica - POSICIONAR, para futura validação por um painel de peritos.

\section{METODOLOGIA}

A existência de instrumentos de apoio à avaliação e à tomada de decisão clínica tem-se revelado uma mais-valia na prática clínica dos profissionais de saúde suportando a decisão e trazendo para a clínica a evidência disponível.

Noutras áreas da clínica, têm sido criadas Mnemónicas [por exemplo a Mnemónica ISBAR utilizada na transmissão verbal para garantir uma comunicação eficaz] para auxiliar a memória, permitindo através de formas simples, memorizar construções complexas, em que cada letra corresponde a um conjunto de intervenções, atividades e/ou procedimentos.

A mnemónica proposta por Baixinho, Ferreira, Rafael e Ferraz, (2016) é um instrumento útil para a promoção da utilização da terapêutica de posição em segurança. No entanto, a multiplicidade de situações clínicas e de desenvolvimento onde pode ser utilizada e a diversidade de aspetos relacionados com a complexidade de cada ser humano dificulta, sintetizar num único instrumento todos os elementos que possam apoiar a decisão clínica (Baixinho, Ferreira, Rafael \& Ferraz, 2016), o que justifica a validação de conteúdo desta mnemónica (Figura 1). 


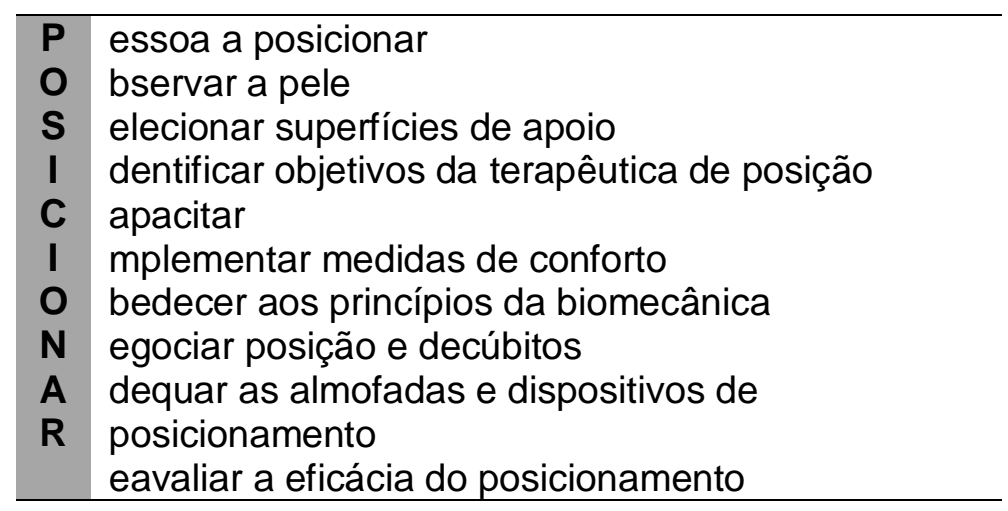

Figura 1. Mnemónica POSICIONAR. Fonte: Baixinho, Ferreira, Rafael \& Ferraz (2016).

Este estudo de abordagem mista tem por meta a validação da mnemónica e compreende três fases, com estudos complementares. A primeira fase correspondeu à revisão da literatura para a construção da Mnemónica (Baixinho, Ferreira, Rafael \& Ferraz, 2016). A segunda fase inclui um estudo qualitativo com consulta a um grupo de peritos para a definição dos indicadores a incluir na mnemónica e cujos resultados apresentamos neste artigo.

$\mathrm{Na}$ terceira fase, os indicadores definidos vão ser submetidos a um painel, utilizando a Técnica de Delphi, para obter consenso entre os peritos, relativamente às proposições apresentadas.

Após a revisão da literatura ter possibilitado a indicação de um conjunto de intervenções a incluir em cada ação determinada pelas letras POSICIONAR, o grupo de investigação concluiu que estas não esgotavam a riqueza da prática clínica e que não se tinha conseguido nem a representatividade, nem a exaustividade para cada ação individualmente e para a mnemónica enquanto um todo. Para colmatar estas dificuldades solicitou-se a colaboração de 9 enfermeiros especialistas em enfermagem e reabilitação, com conhecimento e trabalho desenvolvido nesta área, de forma a obter subsídios.

Os profissionais foram contactados em outubro de 2018, foi-lhes explicado a importância que este instrumento pode ter para a formação e intervenção clínica, a finalidade da sua participação, a duração prevista de resposta e a metodologia. Este contacto prévio serviu ainda para prevenir a abstenção dos participantes.

Esta opção metodológica segue as orientações de autores que observam que a consulta de peritos permite consenso entre especialistas e é útil quando as evidências em uma área são 
limitadas ou contraditórias e as questões são inexploradas ou difíceis de definir (Fletcher \& Marchildon, 2014; Waltz, Strickland \& Lenz, 2016). Por outro lado, outros investigadores argumentam que a obtenção de consenso entre um grupo de indivíduos com experiência num tópico específico é um método particularmente bom de pesquisa (Waltz et al., 2016). Este grupo de peritos não será o mesmo do terceiro estudo, onde pretendemos validar o conteúdo da mnemónica com um painel heterogéneo constituído por clínicos e investigadores.

Foram construídos e enviados dois formulários através do Google Drive®, em momentos diferentes. O primeiro incluía uma única questão em que se solicitava aos colegas que enumerassem TODAS as intervenções associadas ao posicionamento terapêutico, dandose 15 dias úteis para a sua resposta. No corpo do formulário explicou-se a finalidade do estudo e solicitava-se que a resposta fosse desenvolvida para incluir todas as intervenções que os enfermeiros planeiam e executam antes, durante e após o posicionamento terapêutico.

O segundo formulário enviado no mês seguinte solicitava que os profissionais enumerassem as intervenções para cada um dos itens da mnemónica (Pessoa a posicionar; Observação da pele Selecionar superfícies de apoio; Identificar objetivos da terapêutica de posição; Capacitar para o posicionamento; Implementar medidas de conforto; Obedecer aos princípios de biomecânica; Negociar posições e decúbitos; Adequar as almofadas e os dispositivos de posicionamento e Reavaliar a eficácia do posicionamento).

A opção por duas rondas, com estratégias diferentes, teve por intuito na primeira volta explorar as intervenções e na segunda organizá-las por categorias. Concomitantemente, possibilitou que surgissem outras categorias dado que, após a primeira ronda, os profissionais estavam mais despertos no decurso da sua atividade clínica para as 'relembrarem' e identificarem.

As respostas dos participantes foram sujeitas a análise temática de conteúdo segundo Bardin (2011), tendo sido auxiliada pela codificação dos instrumentos e das categorias prédefinidas. Analisaram-se em simultâneo as respostas obtidas na primeira e na segunda ronda.

A opção pela categorização a priori é justificada pelo objetivo do estudo. Na sua formulação obedeceram-se aos critérios de validade, exaustividade e homogeneidade, garantindo que a 
classificação de qualquer elemento do conteúdo deve ser mutuamente exclusiva, tanto para as categorias como para as subcategorias.

O contacto prévio para obter a autorização dos profissionais e o reenvio do instrumento pelos participantes pressupôs a intenção de participação e por esse motivo não foi solicitado a assinatura do termo de consentimento livre e esclarecido. Os participantes foram informados de que as suas respostas eram confidenciais e anónimas e que apenas seriam utilizadas neste âmbito, podendo desistir de participar a qualquer momento, respeitando assim o princípio da Confidencialidade e Anonimato das informações pessoais e do respeito pela vida privada.

\section{RESULTADOS}

Os nove enfermeiros especialistas em enfermagem de reabilitação que colaboraram no estudo, trabalham em média há 21,3 anos. A análise das respostas desses especialistas permitiu definir os indicadores a serem colocados em cada categoria previamente definida (Tabela 1). Os 9 enfermeiros especialistas têm média de idade de 42,4 anos, 55,5\% possuem mestrado e $45,5 \%$ tem curso de especialização em enfermagem de reabilitação.

Tabela 1. Indicadores da Mnemónica POSICIONAR. Lisboa, 2020

\begin{tabular}{llc}
\hline Categoria & \multicolumn{1}{c}{ Sub-Categoria } & $\begin{array}{c}\text { Unidades de } \\
\text { Enumeração }\end{array}$ \\
\hline Pessoa & Individualizar o posicionamento terapêutico em função da condição & 13 \\
a posicionar & clínica e do risco de lesão por pressão; & 11 \\
& Avaliar função músculo esquelética (força muscular, amplitude articular, & 7 \\
& mobilidade e sensibilidade); & 5 \\
& Avaliar estado cognitivo; & 3 \\
& Determinar as preferências da pessoa em relação ao posicionamento; & 2 \\
& Avaliar dor nos diferentes decúbitos; & $\mathbf{4 1}$ \\
\hline & Determinar condições económicas para a aquisição de superfícies de & 14 \\
& apoio. & 8 \\
& Subtotal & 6 \\
\hline Observar a pele e & Avaliar as características da pele na 1a avaliação clínica; & 6 \\
& Avaliar diariamente a pele; & 6 \\
& Avaliar a pele sempre que se verifique alterações clínicas; & 5 \\
& A cada mudança de posicionamento avaliar a pele nas áreas cutâneas & \\
& sobrejacentes às proeminências ósseas; & \\
& O Exame físico da pele deve incluir: cor, humidade, odor, temperatura, & \\
& turgor, textura, integridade e pilosidade. & 6 \\
\hline Selecionar & Subtotal & 5 \\
superfícies & Avaliar necessidade de material de redistribuição de pressão; \\
de apoio & Gerir superfícies de apoio disponíveis; & 1 \\
& Adequar a superfície de apoio à pessoa (risco individual); & $\mathbf{2 4}$
\end{tabular}




\begin{tabular}{|c|c|c|}
\hline Categoria & Sub-Categoria & $\begin{array}{l}\text { Unidades de } \\
\text { Enumeração }\end{array}$ \\
\hline $\begin{array}{l}\text { Identificar } \\
\text { os objetivos da } \\
\text { terapêutica de } \\
\text { posição }\end{array}$ & $\begin{array}{l}\text { Listar as condições de saúde que beneficiam com o uso da terapêutica } \\
\text { de posição; } \\
\text { Antecipar benefícios; } \\
\text { Adequar o posicionamento à condição clínica. } \\
\text { Subtotal }\end{array}$ & $\begin{array}{c}6 \\
4 \\
3 \\
13\end{array}$ \\
\hline Capacitar & $\begin{array}{l}\text { Informar a pessoa sobre o objetivo terapêutico do posicionamento; } \\
\text { Informar a família sobre as finalidades do posicionamento; } \\
\text { Ensinar os cuidadores a posicionar corretamente; } \\
\text { Incentivar o autoposicionamento nas pessoas com independência para } \\
\text { tal; } \\
\text { Treinar a família para dar continuidade a esta terapêutica. } \\
\text { Subtotal }\end{array}$ & $\begin{array}{c}15 \\
10 \\
9 \\
7 \\
5 \\
46\end{array}$ \\
\hline $\begin{array}{l}\text { Implementar } \\
\text { medidas } \\
\text { de conforto }\end{array}$ & $\begin{array}{l}\text { Manter alinhamento corporal; } \\
\text { Garantir que a roupa está 'esticada'; } \\
\text { Manter roupa seca; } \\
\text { Escolher dispositivos de posicionamento confortáveis; } \\
\text { Verificar superfícies de contacto do material com a pele. } \\
\text { Subtotal }\end{array}$ & $\begin{array}{c}8 \\
8 \\
7 \\
4 \\
1 \\
28\end{array}$ \\
\hline $\begin{array}{l}\text { Obedecer } \\
\text { aos princípios } \\
\text { da biomecânica }\end{array}$ & $\begin{array}{l}\text { Manter alinhamento corporal; } \\
\text { Redistribuir adequadamente a pressão; } \\
\text { Respeitar simetria dos hemicorpos nas diferentes posições; } \\
\text { Eliminar forças de fricção no (re)posicionamento } \\
\text { Subtotal }\end{array}$ & $\begin{array}{c}8 \\
7 \\
3 \\
3 \\
21\end{array}$ \\
\hline $\begin{array}{l}\text { Negociar } \\
\text { posição } \\
\text { decúbitos }\end{array}$ & $\begin{array}{l}\text { Identificar posições da preferência da pessoa; } \\
\text { Respeitar posição usual de descanso (dormir); } \\
\text { Determinar capacidade (física) do cuidador para realizar determinados } \\
\text { decúbitos; } \\
\text { Promover a interação social; } \\
\text { Negociar tempo em cada posição. } \\
\text { Subtotal }\end{array}$ & $\begin{array}{c}9 \\
4 \\
2 \\
2 \\
1 \\
18\end{array}$ \\
\hline $\begin{array}{l}\text { Adequar } \\
\text { as almofadas e } \\
\text { os dispositivos } \\
\text { de } \\
\text { posicionamento }\end{array}$ & $\begin{array}{l}\text { Selecionar almofadas que favoreçam a redistribuição da pressão nos } \\
\text { segmentos corporais que estão a apoiar/suportar; } \\
\text { Verificar a adequabilidade (características e segurança) dos materiais; } \\
\text { Adequar às características antropométricas da pessoa; } \\
\text { Garantir que o material é seguro. } \\
\text { Subtotal }\end{array}$ & $\begin{array}{c}7 \\
5 \\
3 \\
1 \\
16\end{array}$ \\
\hline $\begin{array}{l}\text { Reavaliar } \\
\text { a eficácia do } \\
\text { posicionamento }\end{array}$ & $\begin{array}{l}\text { Avaliar pele em contacto com superfície de posicionamento; } \\
\text { Avaliar alivio da sintomatologia (edema, eritema, dispneia, taquicardia, } \\
\text { dor); } \\
\text { Questionar a pessoa quanto ao conforto. } \\
\text { Subtotal } \\
\text { Total }\end{array}$ & $\begin{array}{c}14 \\
12 \\
6 \\
32\end{array}$ \\
\hline
\end{tabular}

Como se percebe pela leitura da tabela emergiram várias intervenções para sustentar o conteúdo dos diferentes itens da mnemónica. As categorias são heterogéneas do ponto de vista das intervenções, número, complexidade e com diferentes unidades de enumeração. 


\section{DISCUSSÃO}

Como referido anteriormente a terapêutica de posição, amplamente usada nos contextos clínicos, é uma área com pouca investigação e com necessidade de mais evidência para suportar a tomada de decisão e a intervenção dos profissionais de saúde que a aplicam. Concordamos com Silva e Nascimento (2012) quando defendem a necessidade de mais estudos acerca deste tema, de forma a visualizar o cuidado de enfermagem sob o ponto de vista clínico, criando evidências que possam baseá-lo num cuidado crítico, prescrito individualmente, conforme a clínica do doente, mesmo na vigência de protocolos.

O desenho da mnemónica POSICIONAR e posterior validação vai contribuir para a existência de um instrumento simples e de rápida aplicação para auxiliar a tomada de decisão clínica e aumentar a segurança na prestação de cuidados.

Como em qualquer área dos cuidados a decisão sobre a implementação desta terapêutica tem de ser tomada pelo profissional de saúde tendo em conta a avaliação da pessoa de acordo com o objetivo do posicionamento, a função, a avaliação da maturidade músculo esquelética e da força muscular, da amplitude articular e da mobilidade, a integração das preferências da pessoa assistida, a avaliação da sua utilização como uma estratégia para o controlo da dor, pela adoção de posições antiálgicas, a promoção da interação social, o risco de úlcera por pressão e de outras potenciais complicações, decorrentes da utilização dos diferentes decúbitos e da própria situação clinica, a avaliação do estado mental, entre outros (Baixinho, Ferreira, Rafael \& Ferraz, 2016; Schutt, Tarver, \& Pezzani, 2018; Santos, Ferreira, \& Baixinho, 2019).

A este respeito Spruce e Van Wicklin (2014) advogam que no caso dos doentes cirúrgicos a sua apreciação deva incluir a avaliação das estruturas circulatória, respiratória, tegumentar, músculo-esquelética e neurológica para garantir que a pessoa é adequadamente posicionada e de modo seguro, prevenindo complicações.

$\mathrm{Na}$ apreciação da pessoa a avaliação da pele assume um papel importante. Esta categoria obteve 39 unidades de enumeração, o que de certa forma é representativo da importância que os profissionais the atribuem, porque uma das principais indicações para 0 (re)posicionamento está associada à prevenção de lesões por pressão, que têm uma elevada prevalência na população idosa, com períodos de imobilidade na cama. Além disso a pele vai estar sempre em contacto ou com a cama, almofadas, ou outro tipo de dispositivo 
auxiliar do posicionamento e consequentemente a sua avaliação vai determinar a própria adequação e a funcionalidade da superfície escolhida em cada contacto estabelecido com a pessoa (NPUAP, EPUAP \& PPPIA, 2014).

$\mathrm{Na}$ identificação dos objetivos os participantes valorizaram listar as condições de saúde que beneficiam com o uso da terapêutica de posição; o antecipar benefícios e a adequação do posicionamento à condição clínica. Tradicionalmente associada à prevenção de úlceras por pressão (Silva \& Nascimento, 2012), esta terapêutica assume-se como crucial para a manutenção da saúde, promoção do conforto, recuperação da doença, prevenção de complicações ao longo de todo o ciclo vital, nomeadamente de lesões músculo-esqueléticas, prevenção da asfixia e prevenção das complicações associadas à imobilidade.

De salientar que as comorbilidades presentes em muitos dos clientes dos cuidados de saúde implicam uma clara antecipação dos benefícios versus malefícios na aplicação desta terapêutica. Por exemplo uma pessoa com dificuldades respiratórias e alto risco de lesão por pressão, em princípio, não poderá efetuar, exclusivamente, as posições descritas pelas associações de referência (NPUAP, EPUAP \& PPPIA, 2014) como as mais eficazes para prevenir as feridas por pressão (semi-dorsal e semi-ventral).

Capacitar $(n=46)$ foi a categoria com maior representatividade, o que aponta para a preocupação dos profissionais em garantir que na sua ausência, por alta hospitalar ou fim da visita domiciliária, a pessoa se tiver potencial físico para se autoposicionar o faça, ou então, que seja auxiliada pelo cuidador.

Esta precaução vai de encontro às recomendações de outros autores que advogam que a pessoa deve ser incentivada a (re)posicionar-se regularmente enquanto estiver deitada ou sentada, sempre que tenha a sua autonomia desenvolvida/preservada, devendo ter acesso a dispositivos de assistência adequados para a promoção da mobilidade (NPUAP, EPUAP \& PPPIA, 2014; Baixinho, Ferreira, Rafael \& Ferraz, 2016).

Outra medida para atingir esta finalidade é a identificação de métodos eficazes de alívio da pressão e educar para a realização dos métodos compatíveis com a capacidade individual, por exemplo ensinar a fazer "elevações" para o alívio da pressão (NPUAP, EPUAP \& PPPIA, 2014). 
O 'O' na mnemónica representa o obedecer aos princípios da biomecânica. $\mathrm{Na}$ análise do discurso escrito dos participantes emerge a manutenção do alinhamento corporal e a redistribuição da pressão, o que vai de encontro a estes princípios (Moore, Cowman, \& Conroy, 2011; Presado et al., 2015). Estudos recentes defendem o alinhamento dos diferentes segmentos corporais mantendo a articulação numa posição 'tão neutra' quanto o possível para diminuir a pressão (Pickenbrock, Ludwig, \& Zapf, 2017).

Consideramos que existe a necessidade de mais investigação que associe os diferentes decúbitos, respeitando a biomecânica com os efeitos na pressão, conforto e prevenção de complicações associadas à manutenção desta terapêutica por longos períodos. Esta recomendação é suportada pelo estudo de Pickenbrock, Zapf, e Dressler (2015) que observam que o posicionamento pode ser feito de maneira convencional ou em 'posição neutra', referindo que esta última opção demonstrou ter melhores efeitos na amplitude de movimento e conforto.

\section{CONCLUSÕES}

A complexidade dos processos de saúde doença que as pessoas vivenciam ao longo do ciclo vital tem orientado os profissionais de saúde para a necessidade de implementar terapêuticas diversificadas e não só farmacológicas, com o intuito de promover ou recuperar a saúde, prevenir complicações decorrentes dos períodos, mais ou menos prolongados de hospitalização e/ou institucionalização e garantir qualidade de vida aos clientes dos cuidados de saúde.

Em termos de prática clinica, importa identificar um conjunto de indicadores que facilitem e permitam sistematizar a avaliação da pessoa e a tomada de decisão dos profissionais, sobre a implementação desta terapêutica.

Este estudo, possibilitou a definição de um conjunto de indicadores para cada uma das ações contidas na Mnemónica POSICIONAR: Pessoa a posicionar; Observação da pele; Selecionar superfícies de apoio; Identificar objetivos da terapêutica de posição; Capacitar para o posicionamento; Implementar medidas de conforto;

Obedecer aos princípios de biomecânica; Negociar posições e decúbitos; Adequar as almofadas e os dispositivos de posicionamento e Reavaliar a eficácia do posicionamento. 
Este é um estudo preliminar para ajudar na definição de um conjunto de indicadores a serem submetidos a avaliação por um painel de peritos. Tem pois limitações associadas ao objetivo do estudo, ao tamanho e às características dos participantes, não podendo ser generalizado. Todavia os resultados são consistentes com a revisão da literatura (efetuada num primeiro momento) e aumentam a consistência do instrumento a enviar ao painel de peritos, só possível com o contributo da abordagem qualitativa.

Terminamos, crentes que o instrumento proposto tem potencialidades para a formação dos profissionais de saúde e para a segurança clínica dos clientes dos cuidados de saúde, e que, nos coloca desafios para a investigação.

Agradecimentos. Agradecemos aos participantes deste estudo o seu contributo para a definição do conteúdo da Mnemónica POSICIONAR.

\section{REFERÊNCIAS}

Baixinho C. L., \& Ferreira, Ó. (2016). Terapêutica de Posição nas transições de vida. In: Lourenço M. J., Ferreira Ó, Baixinho C. L. (Coord.). Terapêutica de Posição: contributo para um cuidado de saúde seguro. Loures: Lusociência; 2016. p.3-13

Baixinho, C. L., Ferreira, Ó., Rafael, H., \& Ferraz, I. (2016). POSICIONAR: uma Mnemónica para a Terapêutica de Posição. In: Lourenço, M. J., Ferreira, Ó, Baixinho, C.L. (2016). Terapêutica de Posição: um contributo para um cuidado de saúde seguro. Loures: Lusodidacta, p.127-137.

Bardin, L. (2011). Análise de Conteúdo. 5ª ed. Lisboa: Edições 70.

Fletcher AJ, Marchildon GP. (2014). Using the Delphi Method for qualitative, participatory action research in health leadership. IJQM, 13(1), 1-18. https://doi.org/10.1177/160940691401300101

Källman, U., Bergstrand, S., Ek, A.-C., Engström, M., Lindberg, L.-G., \& Lindgren, M. (2013). Different lying positions and their effects on tissue blood flow and skin temperature in older adult patients. J Adv Nurs, 69, 133-144. doi:10.1111/j.1365-2648.2012.06000.x

Moore, Z., Cowman, S., \& Conroy, R. M. (2011). A randomized controlled clinical trial of repositioning, using the $30^{\circ}$ tilt, for the prevention of pressure ulcers. $J$ Clin Nurs, 20(17-18), 2633-2644. doi: 10.1111/j.13652702.2011.03736.x. Epub 2011 Jun 27

National Pressure Ulcer Advisory Panel, European Pressure Ulcer Advisory Panel and Pan Pacific Pressure Injury Alliance (NPUAP, EPUAP \& PPPIA). (2014). Prevention and treatment of pressure ulcers: quick reference guide. Perth: Cambridge Media.

Ordem dos Enfermeiros (2013). Cuidados à Pessoa com alterações da Mobilidade - Posicionamentos, transferências e treino de deambulação. Guia Orientador da Prática. Cadernos OE, Série 1, n.․․

Pickenbrock, H. M., Zapf, A., \& Dressler, D. (2015). Effects of therapeutic positioning on vital parameters in patients with central neurological disorders: a randomised controlled trial. $J$ Clin Nurs, 24, 3681-3690. doi:10.1111/jocn.12990 
Pickenbrock, H., Ludwig, V. U., \& Zapf, A. (2017). Support pressure distribution for positioning in neutral versus conventional positioning in the prevention of decubitus ulcers: a pilot study in healthy participants. BMC nurs, 16, 60. https://doi.org/10.1186/s12912-017-0253-z

Presado, M. H. Marques, F. M., Baixinho C. L., Cardoso, M. (2015). Lesões músculo-esqueléticas nos enfermeiros especialistas em saúde maternal: autoperceção dos fatores de risco. In: Costa et al. Atas do $4^{\circ}$ Congresso Ibero-Americano em Investigação Qualitativa e do 6o Simpósio Internacional de Educação e Comunicação: Investigação Qualitativa na saúde, vol 1., pp.193-98.

Santos, L. L., Ferreira, O., \& Baixinho, C. L. (2017). History of therapeutic positioning in nursing care in Portugal (century XIV-XIX). Hist enferm Rev eletrônica,8 (1), 27-35.

Santos, L. L., Ferreira, O., \& Baixinho, C. L. (2019). From the positioning task to the position therapy, a change announced by the history (1900-1953). Hist enferm Rev eletrônica, 10(1), 21-30.

Schutt, S. C., Tarver, C., \& Pezzani, M. (2018). Pilot study: Assessing the effect of continual position monitoring technology on compliance with patient turning protocols. Nurs Open,5,21-28. doi: https://doi.org/10.1002/nop2.105

Silva, R. F., \& Nascimento, M. A. (2012). Mobilização terapêutica como cuidado de enfermagem: evidência surgida da prática. Rev ESC Enferm USP, 46(2), 413-419. doi: https://doi.org/10.1590/S008062342012000200020

Spruce, L., \& Van Wicklin, S.A. (2014). Back to Basics: Positioning the Patient. AORN Journal, 100, $298-305$. doi:10.1016/j.aorn.2014.06.004

Swann J. (2019). Good positioning: the importance of posture. Nurs Res Care, 11(9), 467-9.

Waltz, C., Strickland, O., \& Lenz, E. (2016). Measurement in Nursing and Health Research (5 ${ }^{\text {th }}$. Ed.). New York: Springer Publishing Company. 